\title{
Thermal behaviour and weathering resistance of coil coatings for energy-efficient buildings
}

\author{
Stefano Rossi*, Michele Fedel \\ Department of Industrial Engineering, University of Trento, via Sommarive n. 9, 38123 Trento, Italy
}

\begin{abstract}
In recent years, the aspects of energy-savings in buildings have become increasingly important. Coil coatings are frequently used for roof and façade construction in order to reduce the cost, the building time and the maintenance. In recent years different near infrared (NIR) reflectivity pigments have been developed to increase the capability of paint to reflect the sun's radiation thus minimizing the amount of energy absorbed by the building. Coil coatings containing both traditional and NIR pigments are considered for the assessment of their thermal behaviour. The same painted system produced by three different producers was considered. Using a small scale house model, a W-IR emitting lamp and thermocouples, the amount of energy absorbed by the painted metallic coupons has been evaluated in order to assess the efficiency of the different pigments under investigation. To evaluate effect of ageing, all the different samples were subjected to five accelerated degradation cycles consisting in $168 \mathrm{~h}$ of UV-B irradiation followed by $168 \mathrm{~h}$ of salt spray exposure. The thermal properties of samples were collected. The cool pigments resulted effective to decrease the heat absorbance (difference between standard and NIR paints up to $18^{\circ} \mathrm{C}$ ). The thermal properties were found not to be affected by degradation.
\end{abstract}

\section{Introduction}

Buildings are responsible for approximately $40 \%$ of energy consumption and $36 \%$ of $\mathrm{CO}_{2}$ emissions in the EU [1]. The increasing population in cities and the consequently expansion of urban areas has led to a worsening in the so-called "Urban Heat Island Effect" UHI $[2,3]$.

The annual average air temperature of big cities can be up to $3^{\circ} \mathrm{C}$ warmer than the nearby rural areas during daytime. Heat islands can affect communities by increasing summertime peak energy demand, air conditioning costs, air pollution and greenhouse gas emissions, heat-related illness and mortality [4].

The aspects of energy-savings in buildings have become increasingly important.

A very common way to increase the albedo (the portion of the incident radiation that is reflected by a surface) is to use light colours pigment in order to increase the reflectivity in the visible part of the solar radiation. However, for aesthetically reasons usually is preferable a dark coloured coating, especially for roofs. In this case several solutions are possible.

Near infrared (NIR) reflectivity pigments are frequently inserted into the paints.

In this way a reduction of the heat absorbed by the coating is obtained without modifying its aesthetic properties. The real effectiveness of these paints, however, depends a lot on their colour. This so called cool coatings also show high thermal emittance in order to release the heat as fast as possible to the surrounding environment.

Coil coatings technology consists in the deposition of an organic coating on a metallic (steel or aluminium) plane coils in an industrial plant. Before paint deposition a zinc coating can be applied on steel. Characteristics of this product are the very uniform thickness, the high constant of production, good corrosion protection properties with a limited cost. Thanks to the good cold deformability of this kind of coatings several applications are possible. Coil coatings are frequently used for roof and façade construction in order to reduce the cost, the building time and the maintenance. Considering the final applications, the introduction of NIR pigments in coil coatings could be an interesting solution for the development of a new generation of energy saving buildings. This aspect is not yet widely studied in particular due to the cost of pigments and the difficulties related to the production of samples in small scale, necessary for the research, avoiding influence in the production rate.

In this work, coil coatings containing both traditional and NIR pigments are considered for the assessment of their thermal behaviour. The same painted system produced by three different paints producers was considered.

* Corresponding author: stefano.rossi@unitn.it 


\section{Experimental part}

A hot dip galvanized low carbon steel substrate for coil coating was taken directly from the production line just before the application of the top-coat. The application of the top coat was performed in laboratory by means of a wire-wound coating bar, thus obtaining a wet film thickness of $36 \mu \mathrm{m}$. The curing of the paints was conducted in an electric oven at $350 \mathrm{C}$ for $19 \mathrm{~s}$. A brown colour (RAL 8017) polyester organic coating produced by three different suppliers (called 1,2 and 3) was considered. To highlight the effect of NIR pigments coated sample with the same colour with traditional pigments (with not NIR properties) was considered (figure 1). In all samples rutile pigments were present. The NIR pigments are made of chrome iron hematite. Table 1 reported the studied samples.

Table 1. studied samples.

\begin{tabular}{|c|c|c|}
\hline Sample name & Supplier & NIR pigments \\
\hline 1_NIR & 1 & $\mathrm{x}$ \\
\hline 1_STD & 1 & -- \\
\hline 2_NIR & 2 & $\mathrm{x}$ \\
\hline 2_STD & 2 & -- \\
\hline 3_NIR & 3 & $\mathrm{x}$ \\
\hline
\end{tabular}

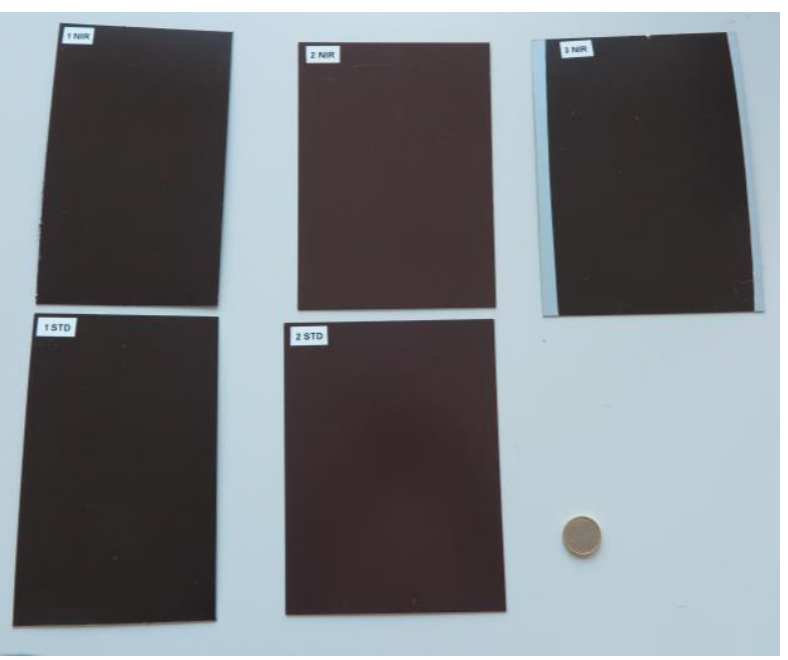

Fig. 1. Studied coated samples.

The first aspect of this research is to individuate an easy method to evaluate the effect of NIR pigments also after weathering of protective system. For this reason, a small scale house model with a plane roof, a W-IR emitting lamp and thermocouples (figure 2) the amount of energy absorbed by the painted metallic coupons has been evaluated in order to assess the efficiency of the different pigments under investigation.
In addition, the temperature distribution of roofs panels has been monitored by using a thermal imaging camera.

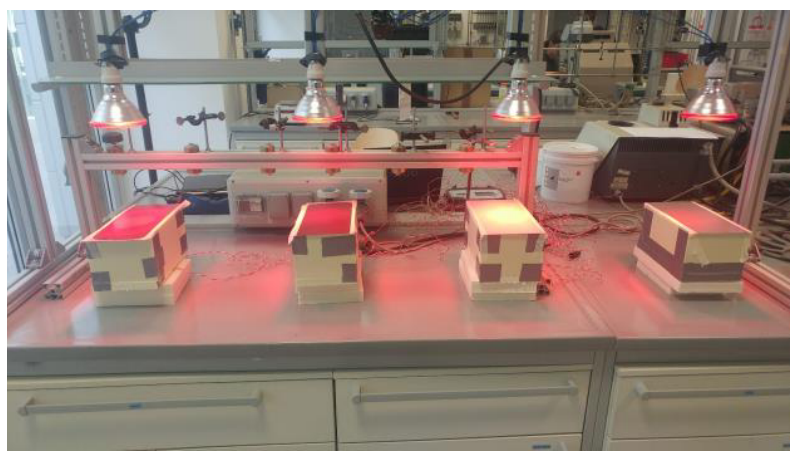

Fig. 2. Picture of the experimental setup. The internal temperature $T_{\text {int }}$ of the small scaled houses and the metal sheet temperature $\mathrm{T}_{\text {sup }}$ were measured in 4 houses simultaneously.

To evaluate effect of ageing of the paint and of the pigments on the thermal behaviour, all the different samples were subjected to 5 accelerated degradation cycles consisting in $168 \mathrm{~h}$ of UV-B irradiation (ASTM G154) followed by $168 \mathrm{~h}$ of salt spray exposure (ASTM $\mathrm{B} 117)$. The total weathering time resulted of $840 \mathrm{~h}$ for UV-B irradiation and $840 \mathrm{~h}$ for salt spray exposure. After each cycle, the thermal properties of samples were collected. To check the change of surface state colour and gloss difference between new and exposed samples are collected.

\section{Results and discussion}

The first point is the verification if the simulate house and the testing set up could permit to obtaining useful information about the coatings' behaviour. In figure 3 the evolution of the temperatures of the rear side of the coated panel and inside the scale house are reported (several repetitions are depicted). 

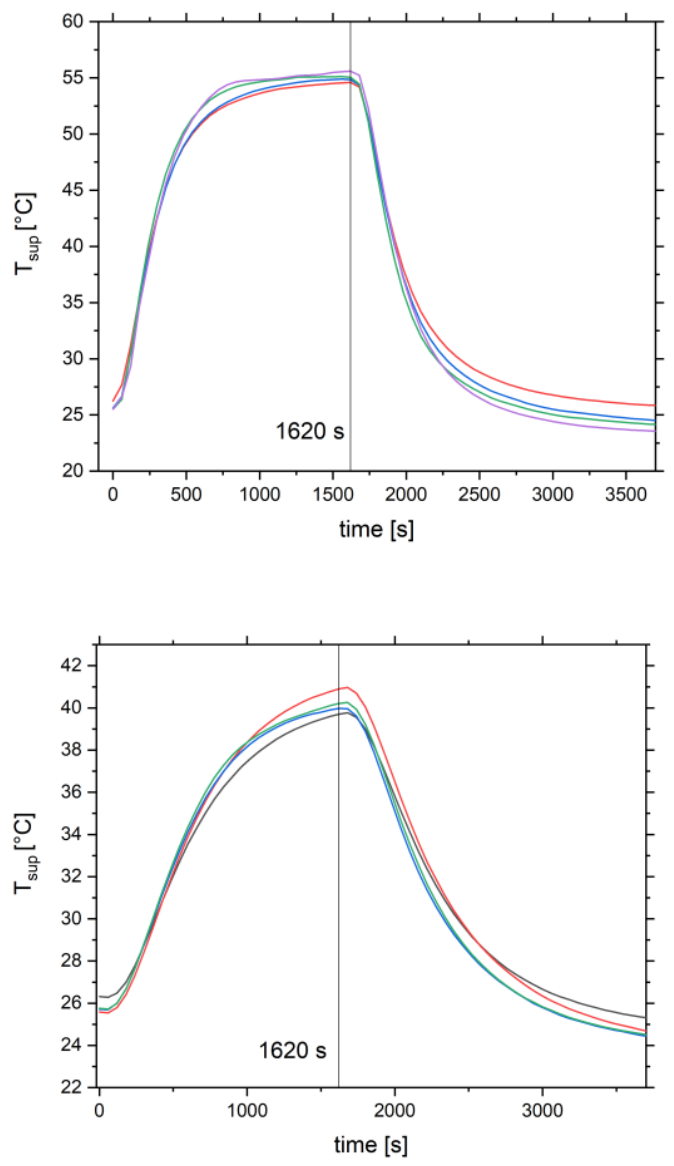

Fig. 3. Temperature of the sample's lower face (up) and inside the small scaled house (down) for 3_NIR samples.

The measured temperature for all tested samples is identical, thus confirming that the set-up allows to obtain repeatable measurements. In addition, considering the temperature trends, after about 1600 second the temperatures reached a stationary state. After that the IR lamps were switched off. Notice that the difference between the surface temperature of the rear part of the panel and that inside the small house is about $15^{\circ} \mathrm{C}$ for the reported sample.

The temperature of the external surface of the panel was also investigated using thermos-camera (Figure 4). In the picture the different coloured area is related to the shielding action of the lamp to avoid the reflection effect during temperature measurement.

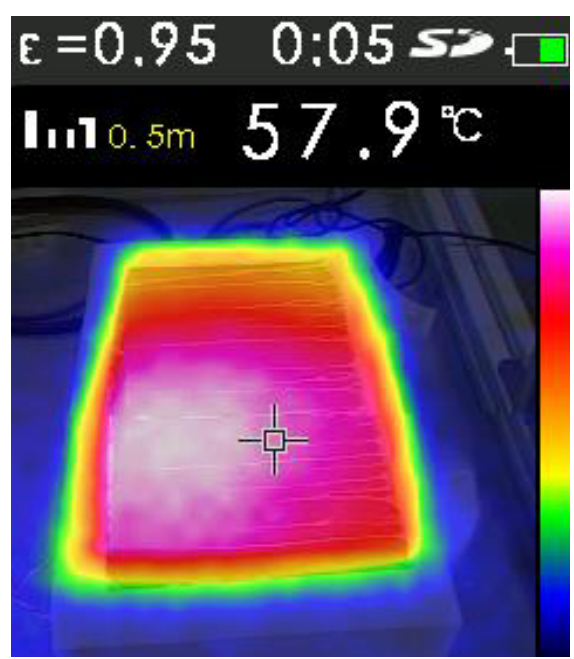

Fig. 4. Thermal image of sample when the IR lamp has just been shielded.

At this point it is possible to characterise the coatings behaviour and the effect of the presence of the NIR pigments.

To gain information on the surface changes during weathering cycles, colour and gloss variations have been monitored. Figures 5 and 6 report the trends of colour and gloss after each cycles.

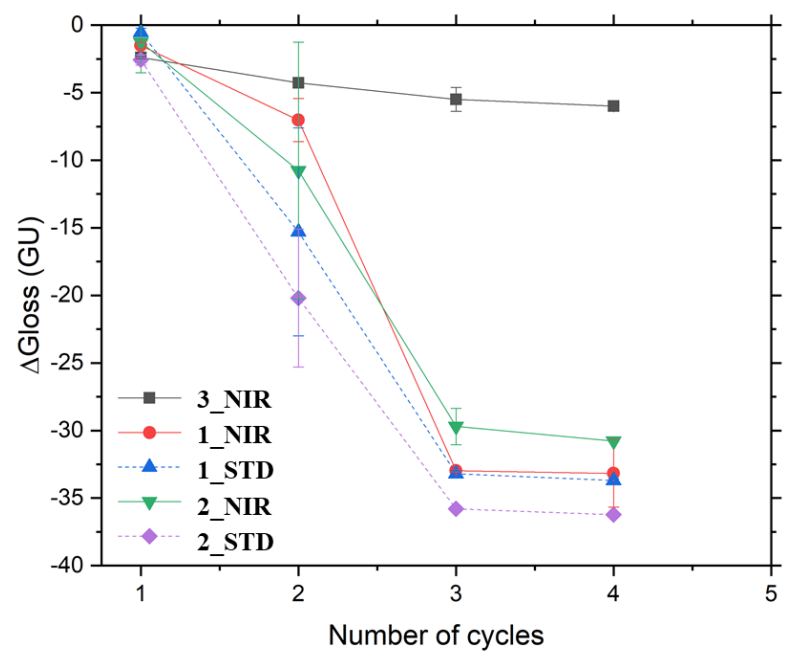

Fig. 5. Gloss evolution as function of the degradation cycles.

Just after 2 cycles all samples, except the 3_NIR shown a sensible reduction of gloss. The trend of the colour variation is pretty different from that of the gloss. It grows uniformly with slopes that depend on the sample.

The colour retention seems not to be related to the colour but depends mainly on the supplier; so, it is probably dependent on the paint formulations and not on the pigments that give the colour at the paint. After 3 or 4 cycles, all samples, except the 1_NIR, present a colour change higher than 2 points, which results visible with the naked ayes.

The thermal properties of the coatings were monitored at the initial state and after each degradation cycle. Figures 7 and 8 show the superficial and internal plateaux temperatures respectively. 


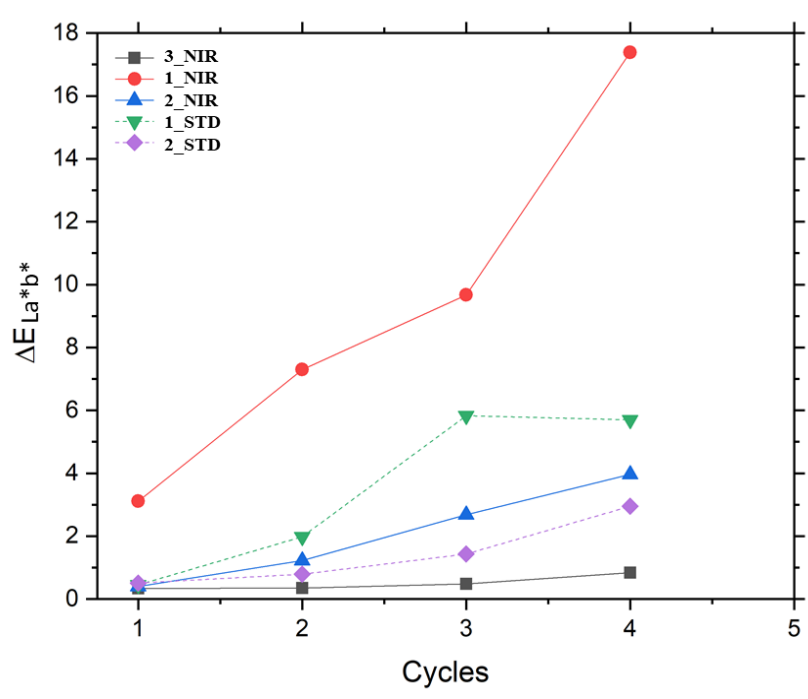

Fig. 6. Colour difference evolution $\triangle E L a * b *$ following CIELab system as function of the degradation cycles.

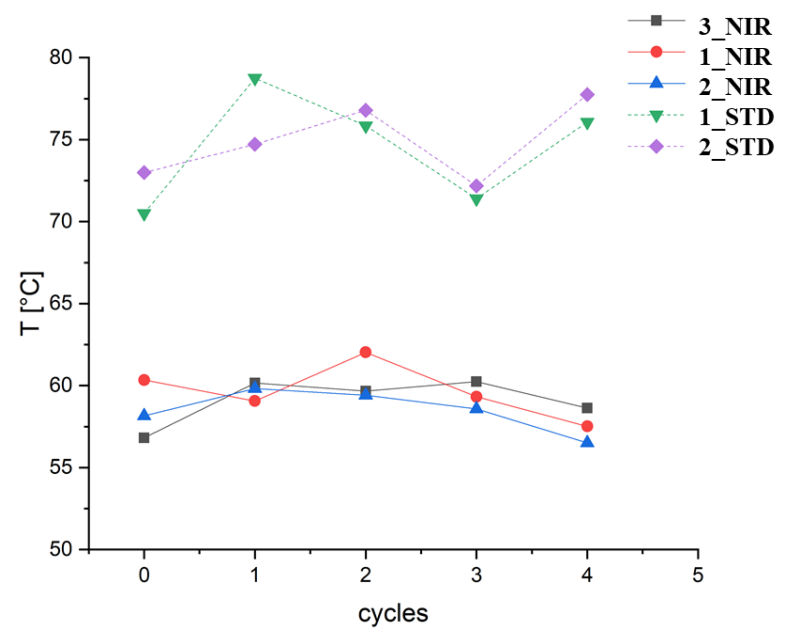

Fig. 7. Superficial temperatures trends.

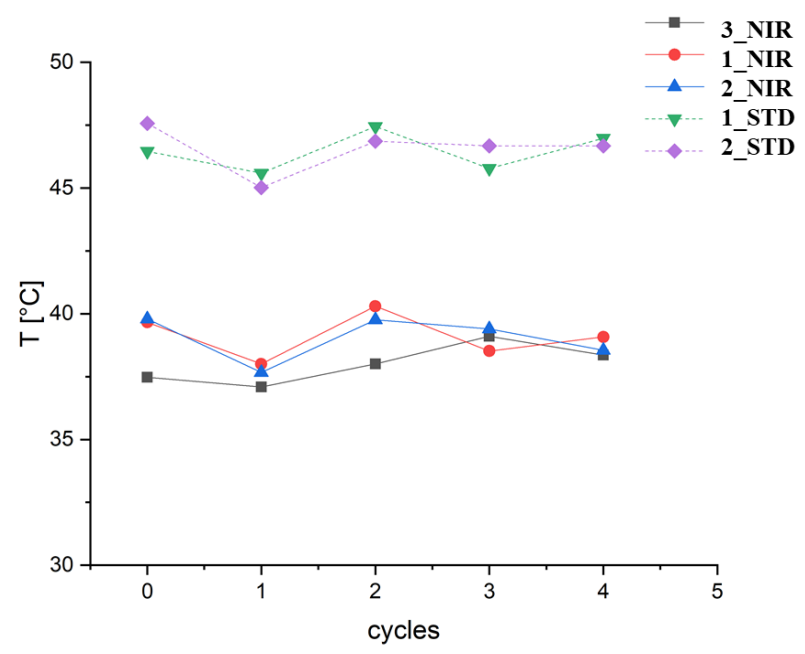

Fig. 8. Internal temperatures trends.

The temperature differences at the initial state between the standard and the NIR reflective products are significant considering superficial and internal temperatures too. The temperature difference between samples both in the NIR reflective version is between $10-15^{\circ} \mathrm{C}$ for the surface temperature and $6.5-7^{\circ} \mathrm{C}$ for the internal one.

The NIR reflective roof panels are effective in reducing the both the superficial and internal temperatures at the initial state. In general, the trend of the NIR reflective roof panels is more stable. The difference between coating with NIR pigments and traditional layers remains also after weathering of organic coatings with gloss and colour changes. No meaningful change of the thermal behaviours of the coatings are produced even though the high change in colour and gloss. Considering this fact, it is possible to affirm that the produced damage is related to the polymeric matrix whereas the pigments remain intact.

\section{Conclusion}

A lab-made set-up allows to measure of the thermal properties of coil coatings with and without NIR pigments.

The change of the thermal behaviour connected with the degradation of paints and appearance change (gloss and colour) was considered.

The cool pigments result effective to decrease the heat absorbance (difference between standard and NIR paints up to $18^{\circ} \mathrm{C}$ ).

The thermal properties were found not to be affected by degradation, made of 5 weathering cycles for a total of $780 \mathrm{~h}$ of UV-B exposure and $780 \mathrm{~h}$ of salt spray test.

The authors are grateful to eng. A. Bonoli from Marcegaglia Carbon Steel (Marina di Ravenna Italy) for samples providing and to Mr D. Dalpiaz for the data collection.

\section{References}

1. European Commission Energy efficiency in buildings, ec.europa.eu/energy/en/topics/energyefficiency/energy-performance-of-buildings (2018).

2. C.A. Konstantinidou, W. Lang, A.M. Papadopoulos, M. Santamouris, Inter. J. Energ. Res. 43, 150 (2019)

3. T. Mavrakou, A. Polydoros, C. Cartalis, M. Santamouris, Climate 16.6, 1 (2018)

4. EPA, Heat Island Effect. www.epa.gov/heat-islands (2017) 